\title{
AUTOMATED POLAR ICE THICKNESS ESTIMATION FROM RADAR IMAGERY
}

\author{
MyAsia A. Reid ${ }^{1}$, Christopher M. Gifford ${ }^{2}$, Michael Jefferson ${ }^{1}$, Eric L. Akers ${ }^{1}$, Gladys Finyom ${ }^{3}$, and Arvin Agah ${ }^{4}$ \\ ${ }^{1}$ Elizabeth City State University, Elizabeth City, NC 27909 \\ ${ }^{2}$ National Security Technology Department, The Johns Hopkins University Applied Physics Laboratory, Laurel, MD 20723 \\ Christopher.Gifford@jhuapl.edu \\ ${ }^{3}$ University of Arizona, Tucson, AZ 85721 \\ ${ }^{4}$ University of Kansas, Lawrence, KS 66045
}

\begin{abstract}
This work focuses on automating the task of estimating Polar ice thickness from airborne radar data acquired over Greenland and Antarctica. This process involves the identification and accurate selection of the ice sheet's surface location and interface between the ice sheet and the underlying bedrock for each measurement. Identifying the surface and bedrock locations in the radar imagery enables the computation of ice sheet thickness, which is important for the study of ice sheets, their volume, and how they may contribute to global climate change. The timeconsuming manual approach requires sparse handselection of surface and bedrock interfaces by several human experts, and interpolating between the selections to save time.
\end{abstract}

Index Terms- Radar Data Processing, Polar Ice Thickness

\section{INTRODUCTION}

Remote sensing methods, such as radar and seismic/acoustic surveys, attempt to acquire data in order to infer properties of the subsurface from a remote location such as the surface, air, or space. A prominent example is the use of radar and acoustic sensors to gather data from the Polar ice sheets, specifically the ice-bedrock interface, from the surface of an ice sheet. Ground and airborne radio-echo sounding of the Greenland and Antarctic ice sheets has been used for many years to determine aspects such as ice thickness, subglacial topography, and mass balance of large bodies of ice. Radar sounding ice sheets is challenging due to the rough surface interface, various stages of melting (both on top of and within the ice sheet), and variation of ice thickness and bedrock topography. Processing the data, including the incorporation of knowledge about the sensing medium, is important for proper interpretation and dissemination of accurate data to the scientific community.

At the University of Kansas, the Center for Remote Sensing of Ice Sheets (CReSIS) [2] performs polar research to gather data and model ice sheets to better understand global climate changes and the possible effects, including sea level rise and human impacts. We have designed, built, and utilized mobile robots to autonomously traverse Polar terrain in Greenland and Antarctica and support radar remote sensing data acquisition. CReSIS has a wide variety of radar data from various radar designs over many years. All of this data is available to the public, providing information such as latitude, longitude, radar travel times, bed echo intensity, and ice thickness.

In this paper, we focus on automating the task of estimating ice thickness from airborne radar data acquired over Greenland and Antarctica. This process involves the identification and accurate selection of the ice sheet's surface location and the interface between the ice sheet and underlying bedrock for each measurement. Identifying the surface and bedrock locations in the radar imagery enables the computation of ice sheet thickness, which is important for the study of ice sheets, their volume, and how they may contribute to global climate change. Previously, this task was performed by several trained human experts, requiring significant resources to complete a single radar data file consisting of thousands of measurements. Given the volume of radar data acquired in the past and its growth each year, automating this task is necessary to provide results to the scientific community in a timely manner.

\section{METHODOLOGY}

Two primary methods have been studied: edge-based, and active contour.

\subsection{Edge detection and following}


In the edge-based approach, edge detection, thresholding, and edge following are utilized to identify the layers of interest for ice thickness estimation. The concept behind this approach is that the surface should be the maximum value in each trace, and that the bedrock reflection layer should be the deepest contiguous layer in the image. Edge detection and thresholding can be used to identify strong edges in the image, which can be further used to locate and follow the layer corresponding to the bottom of the ice sheet. Similar work has been done, in which a skyline detector was implemented by descending down each column and marking pixels as part of the sky until a threshold is exceeded [1].

\subsection{Active contour, cost minimization}

In the minimalist active contour approach, an initial contour is adaptively manipulated and evaluated using image and contour property costs. Motivation for this approach stems from the method utilized onboard the Mars Exploration Rovers (MER) for automatic sky segmentation - via skyline identification - in imagery acquired on the surface of Mars [3][4].In that approach, an active contour consisting of one particle per column, initially located at the top of the image, is pushed downward until it reaches the horizon. The contour arrives at an equilibrium state via the combination of three "forces":

(1) gravity-like force which pushes the contour downward,

(2) upward force based on image edges providing buoyancy, and

(3) tension force between neighboring particles, assuming the horizon's slope does not exceed 45 degrees.

Once the contour is fit to the horizon, the sky can be segmented for further analysis of cloud content (weak layering in the sky). Our approach uses a similar active contour/snake technique to fit a contour to the bottom layer using image and contour costs and a gradient force which pushes the contour upward from the bottom of the image.

\subsection{Ice thickness estimation from radar data}

The contour's shape is adaptively modified and evaluated to minimize path cost or energy through the image to match an image feature, sometimes used for segmenting an image into its cohesive regions. Such approaches have been used in medical imagery (such as MRIs and CAT scans) [5], tracking curves in clutter [6], boundary detection and image segmentation [7].

Results are compared and presented in terms of time requirements, error, and advantages that each method offers. Automatic ice thickness estimation results from 2006 and 2007 Greenland field campaigns show that the edged-based approach offers faster processing (seconds compared to minutes), but suffers from a lack of continuity and smoothness aspects that active contours provide. The active contour approach is more accurate when compared to ground truth selections from human experts, and has proven to be more robust to image artifacts.

Figure 1 shows results from both methods for comparison. Figure 2 shows the human expert selections together with both automated methods for the $20060527 \mathrm{~b}$ (B) flight segment. Each method essentially selects the same surface interface location for the entire file. A zoomed portion of the bedrock is also shown. This allows a direct visual comparison of each method's results, including their differences in certain situations. These figures illustrate where the methods differ and provide examples of situations where they fail to accurately select the bedrock interface. Typically, the edge method is successful for strong contiguous edges in the image, but fails if the bedrock reflection is weak. It also fails when there are artifacts beneath the bedrock. Although faster, this method lacks global continuity and smoothness aspects, which prevents the selection of fully connected contours in some of the images.

\section{CONCLUSION}

The active contour method is more successful in general, with the trade-off of time. It more gracefully bridges weak segments of the bedrock reflection, providing more accurate overall ice thickness estimation for the image. Continuity and stiffness characteristics force the contour to vary smoothly and naturally over bedrock topography. This method can fail in situations where image texture beneath the bedrock is rough, or where strong internal layers are present near the bedrock reflection. Similarly, if the ice is very thin (the bedrock interface is close to the surface), the strong surface reflection is viewed as a regionally lower cost layer to select.

The automated ice thickness estimation technique is now being used by researchers to expedite data processing to release the radar data for public and 
scientific use. Both methods are available and configurable. Current results are significantly speeding up processing of the data. Some radar files, however, are nearly impossible to automatically estimate ice thickness. These challenging files still require human attention. Any image areas that are not adequately identified can be quickly cleaned up by a correction tool that has been developed, in which the user can view the selections, outline those selections to fix, and correct them via interpolation, semi-automated selection using a simple maximum difference method, or manual selection. Users, if time and care are taken to properly complete the task, are superior in terms of being able to quickly determine how to logically bridge gaps in the bedrock reflection and follow faint reflections through heavy noise. This, however, consumes time and human attention that would be more fruitfully used for other efforts.

The research in this paper represents first effort in the goal of fully automated radar image processing and understanding. The described approaches can be advanced for higher level understanding of radar images for science purposes. For example, it is envisioned that using similar automatic processing methods onboard autonomous systems, such as ground or aerial vehicles, can help guide the platform toward areas with specific subsurface characteristics (deep bedrock layer, weak bedrock return, etc.). Furthermore, it can aid in the automatic calibration of the radar's settings for optimal subsurface return intensities using the bedrock return as an optimization parameter during movements of autonomous mobile systems. Currently, automated ice thickness estimation techniques are now being used by researchers to expedite data processing to release the radar data for public and scientific use. Both methods have shown effectiveness and are available to and configurable by the user. One possible extension to this research is to leverage the advantages of both methods. The edge-based method offers speed, while the active contour-based method offers robustness, continuity, and improved accuracy.

Recent results are significantly speeding up processing and dissemination of the data. Some radar files, however, are nearly impossible to automatically estimate ice thickness. These challenging files still require human attention. Utilizing the edge-based method as an initial estimate for the active contour algorithm will likely offer faster convergence and resolve situations where the adaptive contour encounters problems on small artifacts beneath the bedrock echo. Similar methods as those presented in this paper may also be useful for subsurface layer finding/following and segmentation applications for various other mediums. The finding/following, and linking edge fragments are used to construct a connected curve corresponding to one or more salient image features. As subsurface imagery extends further from Earth's surface (high altitude UAVs, space-borne satellites and radar systems, or extending to other planets), automatic characterization such as ice thickness estimation over Greenland and Antarctica ice sheets will be imperative to maximize quality of autonomous science return.

\section{ACKNOWLEDGEMENT}

This material is based upon work supported by the National Science Foundation under Grant No. ANT0424589. Any opinions, findings, and conclusions or recommendations expressed in this material are those of the author (s) and do not necessarily reflect the views of the National Science Foundation.

\section{REFERENCES}

[1] F. Cozman, E. Krotkov, and C. Guestrin, "Outdoor Visual Position Estimation for Planetary Rovers," Autonomous Robots, vol. 9, no. 2, pp. 135-150, 2000.

[2] CReSIS, Center for Remote Sensin of Ice Sheets, 2010 [Online]. Available: http://www.cresis.ku.edu/

[3] V. Gulick, R. Morris, M. Ruzon, and T. Roush, "Autonomous Image Analysis during the 1999 Marsokhod Rover Field Test," Journal of Geophysics Research - Planets, vol. 106, no. E4, pp. 7745-7764, 2000.

[4] T. Roush, M. Shipman, R. Morris, P. Gazis, and L. Pedersen, "Essential Autonomous Science Inference in Rovers (EASIR)," in Proceedings of the IEEE Aerospace Conference, vol. 2, Big Sky, MT, 2004, pp. 790-800.

[5] J. Kratky and J. Kybic, "Three-Dimensional Segmentation of Bones from CT and MRI using Fast Level Sets," in Medical Imaging: Proceeding of the SPIE, vol. 6914, 2008, pp. 691 447-691 447-10.

[6] M. Isard and A. Blake, "Condensation - Conditional Density Propagation for Visual Tracking," International Journal of Computer Vision, vol. 29, no. 1, pp. 5-28, 1998. 
[7] W. Ma and B. S. Manjunath, "EdgeFlow: A

Technique for Boundary Detection and Image

Segmentation," IEEE Transactions on Image Processing, vol. 9, no. 8, pp. 1375-1388, August 2000.
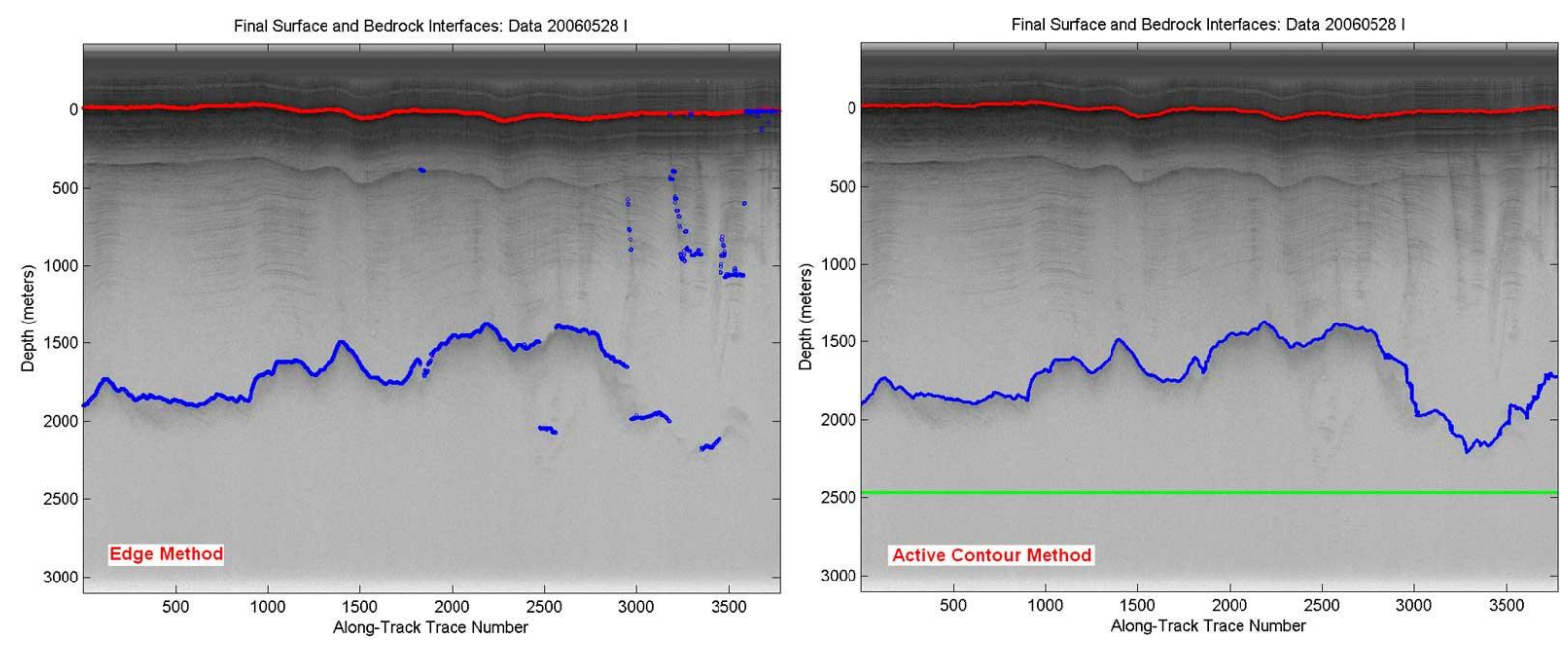

Fig. 1 Comparative results for flight segment 20050528(I). The edge-based method (left) again has difficulty with faint bedrock reflections (e.g., traces 3000, 3250, 3500-3750), but also selects an artifact (e.g., trace 2500) below the bedrock as part of the interface layer. The active contour method (right), due to stiffness and continuity aspects, is able to accurately follow the layer across the faint areas and avoid the artifact.

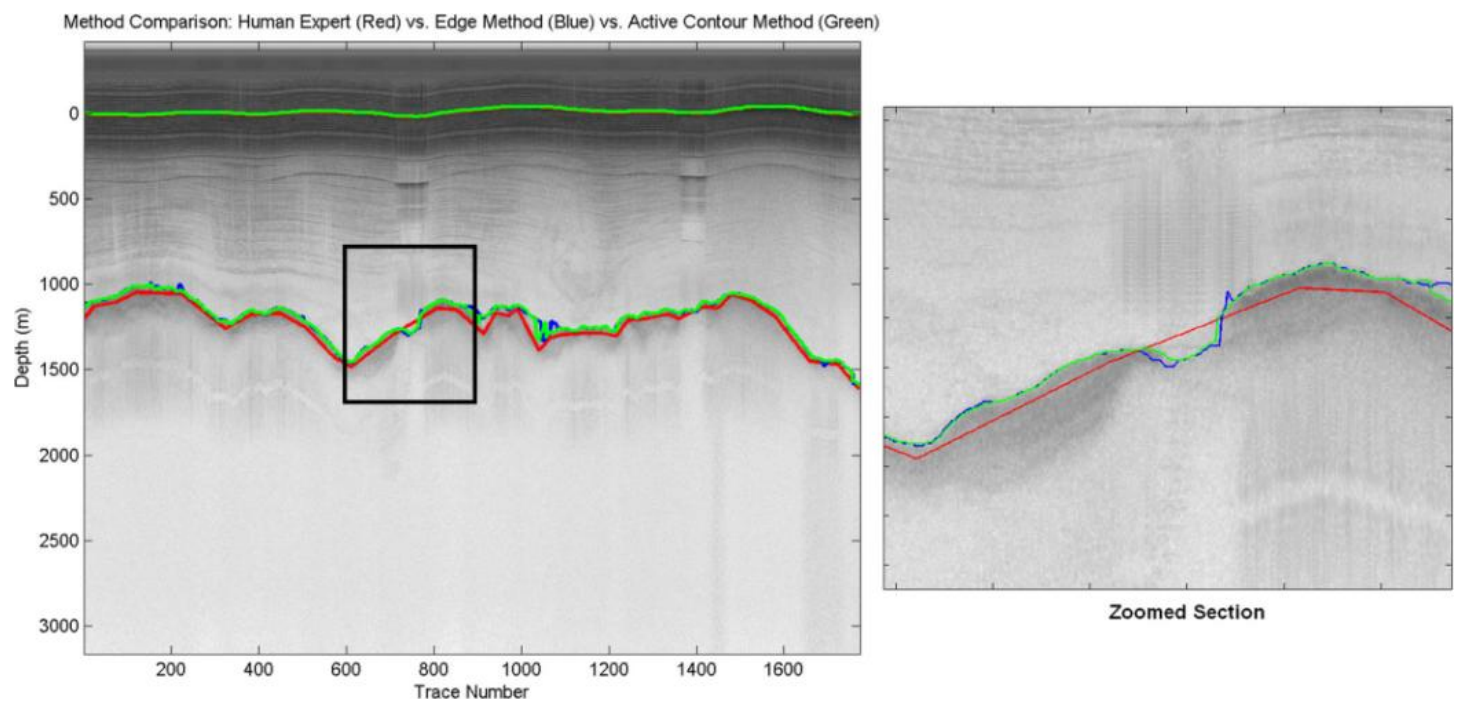

Fig. 2 Comparison of human expert, edge-based method, and active contour method selections for the $20060527 \mathrm{~b}$ (B) flight segment. A zoomed portion of the echogram is displayed for a finer resolution comparative analysis, illustrating the differences between each method for certain situations. 